\title{
Efecto de la suplementación de selenio sobre el rendimiento productivo en cerdos: metaanálisis
}

\author{
Effect of selenium supplementation on productive performance in pigs: \\ meta-analysis
}

\author{
Jimmy Quisirumbay-Gaibor ${ }^{1,2,3}$, Diego Martínez Patiño-Patroni², \\ Carlos Vílchez Perales ${ }^{2}$
}

\section{Resumen}

\begin{abstract}
El objetivo del estudio fue cuantificar el impacto de la suplementación de selenio en la dieta sobre la ganancia diaria de peso, consumo promedio de alimento y eficiencia alimenticia en cerdos. Se ejecutaron 27 metaanálisis a partir de 13 artículos científicos que incluyó un total de 9608 animales. Bajo el modelo de efectos aleatorios se determinó tamaño de efecto y heterogeneidad. Se encontró que la suplementación de selenio mejora la ganancia diaria de peso $(+5.1 \mathrm{~g} /$ día $)$ y eficiencia alimenticia $(+1.5 \mathrm{~g} / \mathrm{kg}$ de alimento). En lechones, la ganancia diaria de peso aumentó en $12.5 \mathrm{~g} /$ día $(\mathrm{p}=0.003)$ y $14.8 \mathrm{~g} / \mathrm{día}$ $(\mathrm{p}=0.007)$ en el análisis general y cuando la fuente fue orgánica, respectivamente. La eficiencia alimenticia en lechones se vio mejorada en $11.7(\mathrm{p}=0.00004), 8.3(\mathrm{p}=0.045)$ y 14.9 $\mathrm{g} / \mathrm{kg}(\mathrm{p}=0.0002)$ en el análisis general, fuente inorgánica y orgánica, respectivamente. Se concluye que la suplementación dietaria de selenio mejora el rendimiento productivo de cerdos, con mayor impacto en lechones y cuando la fuente empleada fue orgánica.
\end{abstract}

Palabras clave: nutrición, alimentación, minerales, inorgánico, orgánico

\section{Abstract}

The aim of this study was to quantify the impact of selenium supplementation in the diet on daily bodyweight gain and feed intake and feed efficiency in pigs. Twenty-seven meta-analyzes were carried out from 13 scientific articles that included a total of 9608 animals. Effect size and heterogeneity were determined under the random effects model.

\footnotetext{
${ }^{1}$ Facultad de Medicina Veterinaria y Zootecnia, Universidad Central del Ecuador, Quito, Ecuador

${ }^{2}$ Escuela de Postgrado, Universidad Nacional Agraria La Molina, Lima, Perú

${ }^{3}$ E-mail: jrquisirumbay@uce.edu.ec
}

Recibido: 31 de marzo de 2019

Aceptado para publicación: 27 de noviembre de 2019

Publicado: 31 de marzo de 2020 
Selenium supplementation was found to improve daily weight gain $(+5.1 \mathrm{~g} /$ day $)$ and feed efficiency $(+1.5 \mathrm{~g} / \mathrm{kg}$ of feed). In piglets, the daily weight gain increased by $12.5 \mathrm{~g} /$ day $(\mathrm{p}=0.003)$ and $14.8 \mathrm{~g} / \mathrm{day}(\mathrm{p}=0.007)$ in the general analysis and when the source was organic, respectively. Piglet feed efficiency was improved by $11.7(\mathrm{p}=0.00004), 8.3(\mathrm{p}=0.045)$ and $14.9 \mathrm{~g} / \mathrm{kg}(\mathrm{p}=0.0002)$ in the general analysis, inorganic and organic source, respectively. It is concluded that dietary selenium supplementation improves the productive performance of pigs, with greater impact on piglets and when the source used was organic.

Key words: nutrition, feeding, minerals, inorganic, organic

\section{INTRODUCCIÓN}

El selenio es un elemento traza esencial que participa en un amplio rango de funciones biológicas para la salud humana y animal. Se ha comprobado su participación en la prevención del cáncer (Tinggi, 2008), disminución de la incidencia de enfermedades cardiovasculares (Benstoem et al., 2015), mejoras en el sistema inmune (Kajander et al., 1991), reducción en las pérdidas por goteo y mejora en la terneza de la carne (Li et al., 2011; Jiang et al., 2017).

Existen dos fuentes de selenio comúnmente usadas en la nutrición animal, denominadas inorgánica (selenito de sodio o selenato) y orgánica, cuya forma es principalmente selenometionina (SeMet) y Se-levadura. El selenito de sodio puede actuar como un prooxidante, el cual es potencialmente tóxico en altos niveles de inclusión en el alimento, mientras que SeMet no presenta este efecto nocivo sobre la salud (Seko et al., 1989; Zhan et al., 2007). Se ha reportado que hay mayor depósito de Se en el tejido muscular cuando la fuente es seleniometionina que cuando es selenio inorgánico (Wang et al., 2011).

Muchos investigadores indican que las levaduras ricas en Se son una forma efectiva de incrementar la actividad de la enzima glutatión peroxidasa (GSH-Px), la concentración de Se tisular y, por lo tanto de mejorar el rendimiento productivo y la calidad de la carcasa en pollos de engorde o en cerdos en crecimiento-finalización (Ortman y Pehrson, 1998; Mahan et al., 1999; Upton et al., 2008; Wang y $\mathrm{Xu}, 2008)$. Las recomendaciones nutricionales por parte del National Research Council (2012) y de la Fundación Española para el Desarrollo de la Nutrición Animal (2013) establecen un nivel de suplementación de selenio entre 0.15 y 0.30 y entre 0.1 y 0.3 $\mathrm{mg} / \mathrm{kg}$ de alimento (ppm), respectivamente, para cerdos. Por otro lado, Rostagno et al. (2017) sugiere niveles de 0.1 a $0.5 \mathrm{mg} / \mathrm{kg}$ (Seinorgánico) y entre 0.07 y $0.23 \mathrm{mg} / \mathrm{kg}$ (Seorgánico).

Valores dentro y fuera de estos rangos han sido utilizados en varios trabajos de investigación con diferentes resultados al evaluar parámetros productivos. El metaanálisis es un método estadístico que resumen y cuantifica el conocimiento adquirido a través del análisis de los resultados de investigaciones ya publicados (Sauvant et al., 2008). Esta herramienta permite obtener una medida del efecto combinado con una mayor precisión que aquella de los estudios individuales y, por lo tanto, tienen una mayor potencia estadística (Catalá-López y Tobías, 2014). El objetivo de este estudio fue cuantificar el impacto de la suplementación dietaria de selenio sobre la ganancia diaria de peso, consumo diario promedio de alimento y eficiencia alimenticia de cerdos a través de un metaanálisis. 


\section{Materiales y Métodos}

\section{Fuente de Información}

Se realizó una búsqueda electrónica de artículos científicos en revistas indexadas con revisión doble ciego en las siguientes bases electrónicas: CAB Direct, Elsevier BiobaseCABS, Google Scholar, MEDLINE, PubMed, Science Direct (Journal), Scopus, Academic Search Complete, CAB Abstract y el Directory of Open Access Journals. Se utilizó una combinación de palabras clave: selenio, Se, dieta, alimento, nutrición, cerdos, lechones, crecimiento, finalización, engorde, así como sus equivalentes en inglés, sin restricciones de fecha.

\section{Criterios de Inclusión}

Se seleccionaron aquellos artículos en los cuales se administró selenio exclusivamente a través de la dieta y con animales libres de enfermedades. Solo fueron admitidos aquellos estudios en los cuales se utilizó selenio hasta un nivel de $0.5 \mathrm{ppm}$. Los artículos debían incluir información respecto al número de sujetos o individuos por unidad experimental y número de unidades experimentales por tratamiento. Los experimentos debían incluir al menos dos tratamientos (incluyendo el grupo control), las fuentes de selenio utilizadas para la suplementación (inorgánica/orgánica), inicio y fin del periodo de estudio y nivel de Se suplementado a través del alimento. Los estudios debían haberse realizado en lechones o en cerdos en fase de crecimiento y/o finalización. Además, debían incluir media (promedio) y alguna medida de variación (desviación estándar (SE) o error estándar (EE)).

\section{Análisis Estadístico}

Para el procesamiento estadístico de los datos se utilizó MIX 2.0 Pro en Microsoft Excel (Bax, 2016). Se determinó el tamaño del efecto de la suplementación de selenio por diferencia de medias (DM) entre el gru- po tratamiento y el control, con intervalos de confianza al 95\%. La heterogeneidad se evaluó por medio del índice de inconsistencia $\left(\mathrm{I}^{2}\right)$ (Cochran, 1954; Higgins y Thompson, 2002). En caso de existir valores altos de heterogeneidad se realizó meta-regresiones con la finalidad de explicar el origen de dicha variabilidad (Borenstein et al., 2011). Se utilizó un modelo de efectos aleatorios según las recomendaciones de Sauvant et al. (2008).

Se ejecutaron 27 metaanálisis a partir de un total de 13 artículos científicos (9608 animales): Mahan y Moxon (1978), Mahan (1985), Mahan y Parrett (1996), MarinGuzman et al. (1997), Lei et al. (1998), Mahan et al. (1999), Mahan y Peters (2004), Tian et al. (2005), Mateo et al. (2007), Li et al. (2011), Speight et al. (2012), Cao et al. (2014), Jlali et al. (2014). Las variables analizadas fueron ganancia diaria de peso (GDP), consumo diario promedio de alimento (CDPA) y eficiencia alimenticia (EA). Para cada variable se realizó un metaanálisis general (sin considerar etapa productiva) y otro considerando la etapa productiva. La etapa productiva fue dividida en dos categorías: (1) lechones y (2) cerdos en crecimiento y finalización. La fuente de selenio fue dividida a su vez en dos grupos: (1) inorgánica (selenito de sodio) y (2) orgánica (selenio-levadura, selenio-metionina, HMSeBA [2-hidroxi-4metilselenobutanoico]).

\section{Resultados}

En el Cuadro 1 se presenta el valor de la media de los parámetros productivos. En el análisis general de la variable GDP se aprecia que los cerdos suplementados con selenio presentan mejores respuestas cuando la fuente suplementada fue la inorgánica. En el análisis por etapa productiva se aprecia que los lechones de los grupos tratados, tanto en el metaanálisis general como cuando las fuentes fueron inorgánica y orgánica, tuvieron mayor GDP. Por el contrario, los cerdos del grupo control en etapa de crecimiento-finalización presentaron mejores GDP. 
Cuadro 1. Resumen variables respuesta para el metaaálisis del impacto de la suplementación alimenticia de selenio sobre la ganancia diaria de peso (GDP), consumo diario promedio de alimento (CDPA) y eficiencia alimenticia (EA) de cerdos

\begin{tabular}{|c|c|c|c|c|c|c|}
\hline \multirow{3}{*}{$\begin{array}{l}\text { Parámetro } \\
\text { productivo }\end{array}$} & \multirow{3}{*}{\multicolumn{2}{|c|}{ Metaanálisis }} & \multicolumn{4}{|c|}{ Resumen variable respuesta } \\
\hline & & & \multicolumn{2}{|c|}{ Tratamiento } & \multicolumn{2}{|c|}{ Control } \\
\hline & & & Media & $\mathrm{DE}$ & Media & $\mathrm{DE}$ \\
\hline \multirow{9}{*}{ GDP (kg/día) } & General & General & 0.703 & 0.203 & 0.704 & 0.210 \\
\hline & & Inorgánica & 0.681 & 0.208 & 0.680 & 0.213 \\
\hline & & Orgánica & 0.726 & 0.199 & 0.727 & 0.205 \\
\hline & Lechones & General & 0.480 & 0.219 & 0.466 & 0.216 \\
\hline & & Inorgánica & 0.485 & 0.208 & 0.472 & 0.204 \\
\hline & & Orgánica & 0.472 & 0.250 & 0.458 & 0.245 \\
\hline & Crecimiento- & General & 0.810 & 0.055 & 0.817 & 0.054 \\
\hline & finalización & Inorgánica & 0.806 & 0.061 & 0.813 & 0.054 \\
\hline & & Orgánica & 0.813 & 0.051 & 0.820 & 0.054 \\
\hline \multirow[t]{9}{*}{ CDPA (kg/día) } & General & General & 1.817 & 0.703 & 1.812 & 0.687 \\
\hline & & Inorgánica & 1.774 & 0.705 & 1.776 & 0.695 \\
\hline & & Orgánica & 1.858 & 0.708 & 1.846 & 0.686 \\
\hline & Lechones & General & 0.998 & 0.652 & 0.985 & 0.645 \\
\hline & & Inorgánica & 1.050 & 0.635 & 1.030 & 0.627 \\
\hline & & Orgánica & 0.929 & 0.708 & 0.926 & 0.701 \\
\hline & Crecimiento- & General & 2.135 & 0.400 & 2.133 & 0.355 \\
\hline & finalización & Inorgánica & 2.122 & 0.414 & 2.134 & 0.364 \\
\hline & & Orgánica & 2.147 & 0.395 & 2.132 & 0.354 \\
\hline \multirow{9}{*}{$\mathrm{EA}(\mathrm{kg} / \mathrm{kg})$} & General & General & 0.427 & 0.086 & 0.424 & 0.083 \\
\hline & & Inorgánica & 0.419 & 0.075 & 0.415 & 0.075 \\
\hline & & Orgánica & 0.434 & 0.095 & 0.431 & 0.090 \\
\hline & Lechones & General & 0.535 & 0.106 & 0.525 & 0.107 \\
\hline & & Inorgánica & 0.501 & 0.106 & 0.496 & 0.111 \\
\hline & & Orgánica & 0.562 & 0.103 & 0.547 & 0.105 \\
\hline & Crecimiento- & General & 0.395 & 0.044 & 0.394 & 0.042 \\
\hline & finalización & Inorgánica & 0.396 & 0.044 & 0.393 & 0.042 \\
\hline & & Orgánica & 0.394 & 0.046 & 0.395 & 0.042 \\
\hline
\end{tabular}

DE: desviación estándar

En el caso del CDPA los cerdos suplementados con selenio presentaron un mayor consumo, excepto en los metaanálisis General-Inorgánica (1.774 vs. $1.776 \mathrm{~kg} /$ día) y Crecimiento-finalización-Inorgánica (2.122 vs. $2.134 \mathrm{~kg} /$ día). Así mismo, los cerdos del grupo suplementado con selenio presentaron valores superiores de EA al compararlos con los cerdos del grupo control, con excepción de los cerdos en crecimiento-finalización su- plementados con la forma orgánica ( 0.394 vs. $0.395 \mathrm{~kg} / \mathrm{kg}$, respectivamente).

El tamaño de efecto expresado en diferencia de medias (MD) muestra que el selenio suplementado en lechones (metaanálisis general) incrementa la GDP en $12.5 \mathrm{~g} / \mathrm{d}$ por encima del grupo control $(\mathrm{p}=0.0037)$ y 14.8 $\mathrm{g} / \mathrm{d}$ cuando la fuente utilizada fue orgánica $(p=0.0071)$ (Cuadro 2). En el caso del CDPA 
Cuadro 2. Tamaño de efecto de la suplementación de selenio en el metaanálisis sobre la ganancia diaria de peso (GDP), consumo diario promedio de alimento (CDPA) y eficiencia alimenticia (EA) de cerdos

\begin{tabular}{|c|c|c|c|c|c|c|}
\hline \multirow{3}{*}{$\begin{array}{l}\begin{array}{l}\text { Parámetro } \\
\text { productivo }\end{array} \\
\text { GDP }\end{array}$} & \multirow{2}{*}{\multicolumn{2}{|c|}{ Metaanálisis }} & \multicolumn{4}{|c|}{ Tamaño de efecto } \\
\hline & & & \multirow{2}{*}{$\frac{\text { MD }}{0.0051}$} & \multicolumn{2}{|c|}{ IC } & \multirow{2}{*}{$\frac{p}{0.0691}$} \\
\hline & \multirow[t]{3}{*}{ General } & General & & -0.0004 & 0.0106 & \\
\hline & & Inorgánica & 0.0024 & -0.0057 & 0.0105 & 0.5600 \\
\hline & & Orgánica & 0.0075 & -0.0001 & 0.0151 & 0.0540 \\
\hline & \multirow[t]{3}{*}{ Lechones } & General & 0.0125 & 0.0041 & 0.0210 & 0.0037 \\
\hline & & Inorgánica & 0.0100 & -0.0037 & 0.0235 & 0.1525 \\
\hline & & Orgánica & 0.0148 & 0.0040 & 0.0256 & 0.0071 \\
\hline & \multirow{3}{*}{$\begin{array}{l}\text { Crecimiento- } \\
\text { finalización }\end{array}$} & General & -0.0032 & -0.0112 & 0.0048 & 0.4340 \\
\hline & & Inorgánica & -0.0058 & -0.0170 & 0.0058 & 0.3282 \\
\hline & & Orgánica & -0.0008 & -0.0119 & 0.0103 & 0.8845 \\
\hline \multirow[t]{9}{*}{ CDPA } & \multirow[t]{3}{*}{ General } & General & 0.0001 & -0.0152 & 0.0154 & 0.9883 \\
\hline & & Inorgánica & -0.0063 & -0.0350 & 0.0225 & 0.6694 \\
\hline & & Orgánica & 0.0036 & -0.0111 & 0.0183 & 0.6322 \\
\hline & \multirow[t]{3}{*}{ Lechones } & General & 0.0137 & -0.0026 & 0.0299 & 0.0988 \\
\hline & & Inorgánica & 0.0324 & 0.0057 & 0.0590 & 0.0172 \\
\hline & & Orgánica & 0.0026 & -0.0178 & 0.0231 & 0.8014 \\
\hline & \multirow{3}{*}{$\begin{array}{l}\text { Crecimiento- } \\
\text { finalización }\end{array}$} & General & -0.0050 & -0.0271 & 0.0172 & 0.6570 \\
\hline & & Inorgánica & -0.0187 & -0.0583 & 0.0209 & 0.3549 \\
\hline & & Orgánica & 0.0055 & -0.0170 & 0.0281 & 0.6302 \\
\hline \multirow[t]{9}{*}{ EA } & \multirow[t]{3}{*}{ General } & General & 0.0015 & -0.0008 & 0.0037 & 0.1944 \\
\hline & & Inorgánica & 0.0006 & -0.0024 & 0.0036 & 0.6839 \\
\hline & & Orgánica & 0.0020 & -0.0013 & 0.0054 & 0.2340 \\
\hline & \multirow[t]{3}{*}{ Lechones } & General & 0.0117 & 0.0061 & 0.0173 & 0.00004 \\
\hline & & Inorgánica & 0.0083 & 0.0002 & 0.0163 & 0.0453 \\
\hline & & Orgánica & 0.0149 & 0.0071 & 0.0226 & 0.0002 \\
\hline & \multirow{3}{*}{$\begin{array}{l}\text { Crecimiento- } \\
\text { finalización }\end{array}$} & General & -0.0003 & -0.0024 & 0.0019 & 0.8040 \\
\hline & & Inorgánica & -0.0006 & -0.0037 & 0.0025 & 0.6968 \\
\hline & & Orgánica & 0.0000 & -0.0031 & 0.0031 & 0.9804 \\
\hline
\end{tabular}

MD: diferencia de medias; IC: intervalo de confianza; p: valor de probabilidad

los lechones que recibieron la fuente inorgánica de Se presentaron un mayor consumo de alimento $(32.4 \mathrm{~g} / \mathrm{d})$ que el grupo control $(\mathrm{p}=0.0172)$, mientras que en la EA se encontró que la suplementación de selenio mejora en lechones general $(\mathrm{p}=0.00004)$ y con la fuente orgánica $(\mathrm{p}=0.0002)$. Es importante destacar que existen varios resultados donde la suplementación de selenio no mejora el parámetro evaluado.
En el Cuadro 3 se muestra los resultados del índice de inconsistencia. Se aprecian valores de moderada heterogeneidad para los metaanálisis: CDPA general-general (28.01\%), CDPA general-inorgánica (57.05\%), CDPA crecimiento-finalizacióngeneral (49.01\%) y CDPA crecimiento-finalización-inorgánica (64.81\%). Todos los demás metaanálisis presentaron valores de baja heterogeneidad $(<25 \%)$ o no la presentaron. 
Cuadro 3. Índice de inconsistencia $\left(\mathrm{I}^{2}\right)$ de la suplementación de selenio sobre la ganancia diaria de peso (GDP), consumo diario promedio de alimento (CDPA) y eficiencia alimenticia (EA) de cerdos

\begin{tabular}{llll}
\hline $\begin{array}{l}\text { Parámetro } \\
\text { productivo }\end{array}$ & & Metaanálisis & $\mathrm{I}^{2}(\%)$ \\
\hline GDP & General & General & 0 \\
& & Inorgánica & 1.29 \\
& & Orgánica & 0 \\
& Lechones & General & 13.01 \\
& & Inorgánica & 21.28 \\
& Crecimiento- & Orgánica & 5.16 \\
& finalización & General & 0 \\
& & Inorgánica & 0 \\
CDPA & General & Orgánica & 0 \\
& & General & 38.01 \\
& & Inorgánica & 57.05 \\
& Lechones & Orgánica & 0 \\
& & General & 0 \\
& & Inorgánica & 0 \\
& Crecimiento- & Orgánica & 0 \\
& finalización & General & 49.01 \\
& & Inorgánica & 64.81 \\
& General & Orgánica & 11.46 \\
& & General & 12.81 \\
& & Inorgánica & 3.25 \\
& Lechones & Orgánica & 20.54 \\
& & General & 0 \\
& & Inorgánica & 0 \\
& Crecimiento- & Orgánica & 0 \\
& & General & 0 \\
& & Inorgánica & 0 \\
& & Orgánica & 2.37 \\
\hline & & &
\end{tabular}

Se hicieron meta-regresiones debido a la moderada heterogeneidad de los valores (Cuadros 4 y 5). El número de repeticiones afecta de manera significativa la variable respuesta para CDPA general-inorgánica y CDPA crecimiento-finalización general e inorgánica. El número de animales por uni- dad experimental y el número de individuos muestreados por unidad experimental también afecta las variables CDPA general-inorgánica y orgánica y CDPA en etapa de crecimientofinalización general e inorgánica. El nivel de inclusión de selenio solo afecta la eficiencia alimenticia general con fuente orgánica. 
Cuadro 4. Meta-regresión para número de repeticiones y número de animales por unidad experimental (UE)

\begin{tabular}{|c|c|c|c|c|c|c|c|c|c|c|}
\hline & \multirow{3}{*}{\multicolumn{2}{|c|}{ Meta-regresión }} & \multicolumn{4}{|c|}{ Número de repeticiones } & \multicolumn{4}{|c|}{ Número de animales por UE } \\
\hline & & & \multicolumn{2}{|c|}{ Intercepto } & \multicolumn{2}{|c|}{ Coef. regresión } & \multicolumn{2}{|c|}{ Intercepto } & \multicolumn{2}{|c|}{ Coef. regresión } \\
\hline & & & Est. & $\mathrm{p}$ & Est. & $\mathrm{p}$ & Est. & $\mathrm{p}$ & Est. & $\mathrm{p}$ \\
\hline \multirow[t]{4}{*}{ CDPA } & General & Inorgánica & -0.054 & 0.009 & 0.010 & 0.006 & -0.067 & 0.004 & 0.012 & 0.003 \\
\hline & & Orgánica & 0.008 & 0.702 & -0.001 & 0.817 & -0.059 & 0.020 & 0.012 & 0.009 \\
\hline & Crec & Gene & -0.008 & $<0.001$ & 0.014 & $<0.001$ & -0.115 & $<0.001$ & 0.021 & $<0.001$ \\
\hline & finalización & Inorgánica & -0.165 & $<0.001$ & 0.028 & $<0.001$ & -0.151 & $<0.001$ & 0.024 & 0.005 \\
\hline EA & General & Orgánica & -0.006 & 0.209 & 0.001 & 0.073 & 0.006 & 0.307 & -0.001 & 0.474 \\
\hline
\end{tabular}

Est: Estimado; p: valor de probabilidad

Cuadro 5. Meta-regresión para número de individuos muestreados por unidad experimental (UE) y nivel de selenio

\begin{tabular}{|c|c|c|c|c|c|c|c|c|c|c|}
\hline & \multirow{3}{*}{\multicolumn{2}{|c|}{ Meta-regresión }} & \multicolumn{4}{|c|}{$\begin{array}{l}\text { Número de individuos } \\
\text { muestreados por UE }\end{array}$} & \multicolumn{4}{|c|}{ Nivel de selenio } \\
\hline & & & \multicolumn{2}{|c|}{ Intercepto } & \multicolumn{2}{|c|}{ Coef. regresión } & \multicolumn{2}{|c|}{ Intercepto } & \multicolumn{2}{|c|}{ Coef. regresión } \\
\hline & & & Est. & $\mathrm{p}$ & Est. & $\mathrm{p}$ & Est. & $\mathrm{p}$ & Est. & $\mathrm{p}$ \\
\hline \multirow[t]{4}{*}{ CDPA } & General & Inorgánica & -0.067 & 0.004 & 0.012 & 0.003 & -0.032 & 0.095 & 0.128 & 0.078 \\
\hline & & Orgánica & -0.060 & 0.020 & 0.012 & 0.010 & 0.008 & 0.612 & -0.016 & 0.754 \\
\hline & Crecimiento- & General & -0.115 & $<0.001$ & 0.021 & $<0.001$ & -0.022 & 0.168 & 0.006 & 0.395 \\
\hline & finalización & Inorgánica & -0.151 & $<0.001$ & 0.024 & 0.005 & -0.059 & 0.011 & 0.146 & 0.120 \\
\hline EA & General & Orgánica & 0.006 & 0.307 & -0.001 & 0.474 & -0.003 & 0.287 & 0.022 & 0.039 \\
\hline
\end{tabular}

Est: Estimado; p: valor de probabilidad

\section{Discusión}

El metaanálisis muestra que la suplementación de selenio incrementa la ganancia diaria de peso y la eficiencia alimenticia de manera general, así como en lechones en particular, independientemente de la fuente de selenio. Los lechones que reciben una suplementación de selenio ganan $12.5 \mathrm{~g}$ más al día $(p=0,0037)$ en comparación con el grupo control. Los lechones con fuente orgánica de selenio ganan $14.8 \mathrm{~g}$ adicionales al día $(\mathrm{p}=0.0071)$ frente al grupo control, pudiendo llegar a ganar hasta $26.5 \mathrm{~g} / \mathrm{d}$ más que el gru- po control, según el límite superior del intervalo de confianza.

Los lechones que recibieron el selenio inorgánico consumieron $32.4 \mathrm{~g}$ más de alimento que el grupo control ( $\mathrm{p}=0.0171)$. Así mismo, se destaca la eficiencia alimenticia de los lechones, la cual aumenta con la suplementación de selenio en 11.7 $(p=0.00004), 8.3(p=0.045)$ y $14.9 \mathrm{~g}$ $(\mathrm{p}=0.0002)$ que en el lechón no suplementado (control) para el análisis general, fuente inorgánica y orgánica, respectivamente. La suplementación de selenio orgánico en la etapa de crecimiento y finalización incrementa 
en $5.5 \mathrm{~g} / \mathrm{d}$ el consumo de alimento $\mathrm{g} / \mathrm{d}$ $(\mathrm{p}=0.63)$. Por lo tanto, estos resultados indican claramente que la suplementación de este mineral tiene mayor impacto en lechones.

Los efectos positivos del selenio son logrados debido a que este mineral permite un adecuado funcionamiento de las seleno-proteínas. Estas proteínas incluyen a la enzima antioxidante glutatión peroxidasa (GSH-Px) (Rederstorff et al., 2006; Pappas et al., 2008), las cuales tienen un importante rol en la defensa antioxidante celular, respuesta inmune y reducción de la inflamación, destinando los nutrientes ingeridos a través del alimento hacia el depósito tisular y la consecuente ganancia de peso (Arthur et al., 2003; Schomburg et al., 2004). En cuanto a la fuente de selenio, se aprecia claramente que la fuente orgánica mejora todos los parámetros productivos evaluados, debido posiblemente a su mayor biodisponibilidad en comparación con la fuente inorgánica (Zhan et al., 2007).

Los resultados encontrados en el presente estudio confirman el efecto positivo de la inclusión de selenio sobre el desempeño productivo de los cerdos. Aaron y Hays (2004) destacan la importancia del número adecuado de repeticiones por tratamiento o grupo en los estudios experimentales en cerdos. En el presente trabajo de investigación se observa como el número de repeticiones afecta la variable respuesta consumo diario promedio de alimento. Por lo tanto, estos factores deberán ser tomados en cuentan al momento de analizar las variables consumo y eficiencia en futuros trabajos de investigación.

\section{Conclusiones}

- La suplementación dietaria de selenio mejora el rendimiento productivo (ganancia de peso y eficiencia alimentaria) de cerdos.

- El beneficio es mayor en lechones y cuando la fuente de selenio es orgánica.

\section{Literatura Citada}

1. Aron D K, Hays VW. 2004. How many pigs? Statistical power considerations in swine nutritional experiments. J Anim Sci 82: 245-254. doi: 10.2527/2004.8213_supplE245x

2. Arthur JR, McKenzie RC, Beckett GJ. 2003. Selenium in the immune system. J Nutr 7: 1457S-1459S. doi: 10.1093/jn/ 133.5.1457S

3. Bax L. 2016. MIX 2.0 - Professional software for meta-analysis in Excel v. 2.0.1.5. BiostatXL. [Internet]. Disponible en: https://www.meta-analysis-madeeasy.com/

4. Benstoem C, Goetzenich A, Kraemer $S$, Borosch S, Manzanares W, Hardy G, Stoppe C. 2015. Selenium and its supplementation in cardiovascular disease-what do we know? Nutrients 7: 3094-3118. doi: 10.3390/nu7053094

5. Borenstein M, Hedges LV, Higgins JP, Rothstein HR. 2011. Introduction to meta-analysis. Chichester: John Wiley \& Sons. $187 \mathrm{p}$.

6. Cao J, Guo F, Zhang L, Dong B, Gong L. 2014. Effects of dietary selenomethionine supplementation on growth performance, antioxidant status, plasma selenium concentration, and immune function in weaning pigs. J Anim Sci Biotechno 5: 46. doi: 10.1186/2049-1891-5-46

7. Catalá-López F, Tobías A. 2014. Metaanálisis en ensayos clínicos aleatorizados, heterogeneidad e intervalos de predicción. Med Clin-Barcelona 142: 270-274. doi: 10.1016/j.medcli.2013.06.013

8. Cochran WG. 1954. The combination of estimates from different experiments. Biometrics 10: 101-129. doi: 10.2307/ 3001666

9. [FEDNA] Fundación Española para el Desarrollo de la Nutrición Animal. 2013. Necesidades nutricionales para ganado porcino. Normas FEDNA. $2^{\circ}$ ed. España: FEDNA. 40 p. 
10. Higgins JP, Thompson SG. 2002. Quantifying heterogeneity in a meta analysis. Stat Med 21: 1539-1558. doi: $10.1002 / \operatorname{sim} .1186$

11. Jiang J, Tang X, Xue Y, Lin G, Xiong YL. 2017. Dietary linseed oil supplemented with organic selenium improved the fatty acid nutritional profile, muscular selenium deposition, water retention, and tenderness of fresh pork. Meat Sci 131: 99-106. doi: 10.1016/ j.meatsci.2017.03.014

12. Jlali M, Briens M, Rouffineau F, Geraert PA, Mercier Y. 2014. Evaluation of the efficacy of 2-hydroxy4-methylselenobutanoic acid on growth performance and tissue selenium retention in growing pigs. J Anim Sci 92: 182-188. doi: 10.2527/jas.2013-6783

13. Kajander EO, Harvima RJ, Eloranta TO, Martikainen H, Kantola M, Kärenlampi SO, Akerman K. 1991. Metabolism, cellular actions, and cytotoxicity of selenomethionine in cultured cells. Biol Trace Elem Res 28: 57-68. doi: 10.1007/bf02990463

14. Lei XG, Dann HM, Ross DA, Cheng WH, Combs GF, Roneker KR. 1998. Dietary selenium supplementation is required to support full expression of three selenium-dependent glutathione peroxidases in various tissues of weanling pigs. J Nutr 128: 130-135. doi: $10.1093 / \mathrm{jn} / 128.1 .130$

15. Li JG, Zhou JC, Zhao H, Lei XG, Xia XJ, Gao G, Wang KN. 2011. Enhanced water-holding capacity of meat was associated with increased Sepw1 gene expression in pigs fed selenium-enriched yeast. Meat Sci 87: 95-100. doi: 10.1016/ j.meatsci.2010.05.019

16. Mahan DC. 1985. Effect of inorganic selenium supplementation on selenium retention in postweaning swine. J Anim Sci 61: 173-178. doi: 10.2527/ jas $1985.611173 \mathrm{x}$

17. Mahan DC, Cline TR, Richert B. 1999. Effects of dietary levels of selenium-enriched yeast and sodium selenite as selenium sources fed to growing-finishing pigs on performance, tissue selenium, serum glutathione peroxidase activity, carcass characteristics, and loin quality. J Anim Sci 77: 2172-2179. doi: 10.2527/1999.7782172x

18. Mahan DC, Moxon AL. 1978. Effects of adding inorganic or organic selenium sources to the diets of young swine. J Anim Sci 47: 456-466. doi: 10.2527/ jas1978.472456x

19. Mahan DC, Parrett NA. 1996. Evaluating the efficacy of seleniumenriched yeast and sodium selenite on tissue selenium retention and serum glutathione peroxidase activity in grower and finisher swine. J Anim Sci 74: 29672974. doi: $10.2527 / 1996.74122967 \mathrm{x}$

20. Mahan DC, Peters JC. 2004. Longterm effects of dietary organic and inorganic selenium sources and levels on reproducing sows and their progeny. $\mathrm{J}$ Anim Sci 82: 1343-1358. doi: 10.2527/ 2004.8251343x

21. Marin-Guzman J, Mahan DC, Chung YK, Pate JL, Pope WF. 1997. Effects of dietary selenium and vitamin E on boar performance and tissue responses, semen quality, and subsequent fertilization rates in mature gilts. J Anim Sci 75: 29943003. doi: 10.2527/1997.75112994x

22. Mateo RD, Spallholz JE, Elder R, Yoon I, Kim SW. 2007. Efficacy of dietary selenium sources on growth and carcass characteristics of growingfinishing pigs fed diets containing high endogenous selenium. J Anim Sci 85: 1177-1183. doi: $10.2527 /$ jas.2006-067

23. Miller WT, Schoening HW. 1938. Toxicity of selenium fed to swine in the form of sodium selenite. J Agric Res 56: 831-842.

24. [NRC] National Research Council. 2012. Nutrient requirements of swine. $11^{\text {th }}$ ed. Washington: National Academies Press. 86 p.

25. Ortman K, Pehrson B. 1998. Selenite and selenium yeast as feed supplements to growing fattening pigs. J Vet Med A 45: 551-557. doi: 10.1111/j.14390442.1998.tb00859.x 
26. Pappas AC, Zoidis E, Surai PF, Zervas G. 2008. Selenoproteins and maternal nutrition. Comp Biochem Phys B 151:361-372. doi: 10.1016/j.cbpb.2008.08.009

27. Rederstorff M, Krol A, Lescure A. 2006. Understanding the importance of selenium and selenoproteins in muscle function. Cell Mol Life Sci 63: 52-59. doi: 10.1007/s00018-005-5313-y

28. Rostagno HS, Teixeira LF, Hannas MI, Lopes J, Sakomura NK, Perazzo FG, Saraiva A, Teixeira ML, et al. 2017. Tablas brasileñas para aves y cerdos. $4^{\circ}$ ed. Viçosa: Universidad Federal de Viçosa. $444 \mathrm{p}$.

29. Sauvant D, Schmidely P, Daudin JJ, St-Pierre NR. 2008. Meta-analyses of experimental data in animal nutrition. Animal 2: 1203-1214. doi: 10.1017/ S1751731108002280

30. Schomburg L, Schweizer U, Köhrle J. 2004. Selenium and selenoproteins in mammals: extraordinary, essential, enigmatic. Cell Mol Life Sci 61: 19881995. doi: 10.1007/s00018-004-4114-z

31. Seko Y, Saito, Y, Kitahara J, Imura N. 1989. Active oxygen generation by the reaction of selenite with reduced glutathione in vitro. In: Selenium in biology and medicine. Berlin: Springer. p 70-73.

32. Speight SM, Estienne MJ, Harper AF, Barb CR, Pringle TD. 2012. Effects of organic selenium supplementation on growth performance, carcass measurements, tissue selenium concentrations, characteristics of reproductive organs, and testis gene expression profiles in boars. J Anim Sci 90: 533-542. doi: 10.2527/jas.2010-3747
33. Tian JZ, Yun MS, Kong CS, Piao LG, Long HF, Kim JH, Han IK. 2005. Effects of different products and levels of selenium on growth, nutrient digestibility and selenium retention of growing-finishing pigs. Asian Austral J Anim Sci 19: 61-66. doi: 10.5713/ ajas. 2006.61

34. Tinggi $U$. 2008. Selenium: its role as antioxidant in human health. Environ Health Prev 13: 102-108. doi: 10.1007/ s12199-007-0019-4

35. Upton JR, Edens FW, Ferket PR. 2008. Selenium yeast effect on broiler performance. Int J Poult Sci 7: 798-805. doi: 10.3923/ijps.2008.798.805

36. Wang $Y B, X u$ BH. 2008. Effect of different selenium source (sodium selenite and selenium yeast) on broiler chickens. Anim Feed Sci Tech 144: 306314. doi: 10.1016/j.anifeedsci.2007.10.012

37. Wang YX, Zhan XA, Yuan D, Zhang $X W, W u$ RJ. 2011. Effects of selenomethionine and sodium selenite supplementation on meat quality, selenium distribution and antioxidant status in broilers. Czech J Anim Sci 56: 305-313. doi: 10.17221/1296-CJAS

38. Zarczynska K, Sobiech P, Radwinska $J W$. 2013. Effects of selenium on animal health. J Elementol 18: 329-340. doi: 10.5601/jelem.2013.18.2.12

39. Zhan X, Wang M, Zhao R, Li W, Xu Z. 2007. Effects of different selenium source on selenium distribution, loin quality and antioxidant status in finishing pigs. Anim Feed Sci Tech 132: 202-211. doi: 10.1016/j.anifeedsci.2006.03.020 